\title{
Una intervención multidisciplinaria domiciliaria redujo las internaciones en pacientes con insuficiencia cardíaca
}

Effects of a multidisciplinary home-based intervention on unplanned readmissions and survival among patients with chronic congestive heart failure: a randomized controlled study. Stewart S, Marley J, Horowitz J. Lancet 1999;354:1077-83.

\section{Objetivo}

Evaluar si una intervención domiciliaria (ID) realizada por enfermeros puede reducir las muertes extrahospitalarias y las reinternaciones no programadas.

\section{Diseño}

Estudio aleatorizado controlado, seguimiento a 6 meses.

Lugar

Hospital de derivación, Adelaide, Australia.

\section{Pacientes}

Se incluyeron 200 pacientes, mayores de 55 años (edad promedio 76), $62 \%$ hombres. Los pacientes eran incluidos al alta hospitalaria de internación por insuficiencia cardíaca congestiva (ICC) descompensada. Se excluyeron los pacientes con isquemia extensa reversible como precipitante de la ICC, enfermedad valvular con necesidad de tratamiento quirúrgico, plan de transplante cardíaco, y residencia fuera del área de influencia. El seguimiento fue completo.

\section{Intervención}

Cien pacientes fueron asignados a cuidado habitual: visita con médico de cabecera a las 2 semanas, normas de planificación del alta que incluía contacto previo al alta y ambulatorio con enfermera de rehabilitación cardíaca, dietista, trabajadora social, farmacéutico y enfermera comunitaria según necesidad. Otros 100 pacientes fueron asignados a ID que incluía una visita estructurada a domicilio por una enfermera cardiológica a los 7 a 14 días del alta. En esta visita se realizaba: examen físico, evaluación de adherencia al tratamiento, evaluación de la capacidad de reconocer los signos y síntomas de descompensación por parte de los pacientes, evaluación de la ingesta de fluidos y sodio, actividad física, red de soporte psicosocial y uso de recursos comunitarios. La enfermera enviaba un informe de sus hallazgos al médico de cabecera y al cardiólogo. En base a esta evaluación los pacientes recibían consejos sobre medicación, adherencia al tratamiento, ejercicios y monitoreo del peso diario. La visita domiciliaria era repetida si se producían 2 o más internaciones no planeadas en 6 meses. Los pacientes eran contactados telefónicamente cada 3 meses.

\section{Medición de resultados principales}

El punto final primario del estudio fue combinado*: La frecuencia de internaciones no planeadas más las mortalidad dentro de los seis meses.

\section{Resultados principales}

De los 100 pacientes asignados a ID, 88 recibieron la visita (2 fallecieron antes de la visita y 10 retiraron su consentimiento). La mediana de duración de la visita fue de dos horas. A seis meses, el grupo ID presentó: 1) menor incidencia del punto final primario combinado* en relación al grupo control: 77 vs. $129, p=0.02 ; 2$ ) menos internaciones no planeadas: 68 vs. $118, p=0.03$; y 3) menos días de estadía hospitalaria: 460 vs. 1174 días, $p=0.01$. La mortalidad fue similar en ambos grupos: ID 9\%, control 11\%, $p=0.64$. El grupo ID presentó una tendencia a menores costos hospitalarios ( $900 \$$ vs $2200 \$, p=N S$ ). Los costos a mbulatorios fueron similares. A 3 meses de la inclusión, los pacientes del grupo ID presentaron mejoria en la calidad de vida, pero no hubo diferencias a los 6 meses.

\section{Conclusiones}

En pacientes con ICC luego del alta hospitalaria, una intervención basada en una visita a domicilio por personal de enfermería redujo las internaciones no planeadas y el número de días de estadía hospitalaria.

\section{COMENTARIO}

A pesar del desarrollo de tratamientos efectivos para la ICC, su pronóstico continúa siendo pobre. La prevalencia de ICC está en aumento, y se estima que el $2 \%$ de la población general la padece. En Estados Unidos, la ICC es la principal causa de internación en mayores de 65 años, y es la enfermedad cardiovascular más costosa, en general debido a las reinternaciones. ${ }^{1}$ Muchas reinternaciones se producen en los primeros días del alta hospitalaria, y la mayoría obedece a falta de adherencia con la medicación o la dieta hiposódica, y en menor medida a eventos cardiovasculares o progresión de la enfermedad. Muchos pacientes con ICC presentan otras comorbilidades, están polimedicados, presentan falta de red social, problemas financieros y transtornos cognitivos. La dificultad en el manejo de estos pacientes, en particular en clase funcional avanzada, dió lugar a la evaluación de diferentes intervenciones multidisciplinarias para su mejor manejo. Se han publicado 10 estudios no aleatorizados y 11 aleatorizados sobre diferentes programas de manejo (disease management) en ICC. En estos estudios (que incluyen diferentes intervenciones: enfermeras, farmacéuticos, asistentes sociales, etc) se observaron resultados consistentes en relación a la reducción en las reinternaciones, mejoría en la calidad de vida y la satisfacción de pacientes, y en algunos, menores costos.

En este estudio, el $40 \%$ de los pacientes ya presentaba signos de descompensación en la visita, $25 \%$ no cumplía con la medicación y $90 \%$ no conocía la importancia de la dieta. Estos hallazgos demuestran que el manejo intrahospitalario no prepara a los pacientes para un cuidado adecuado luego del alta. El grupo control recibía también consejos previos al alta, y acceso fácil a personal de salud no médico según necesidad, lo que podría haber subestimado el efecto de la intervención. El $10 \%$ de los pacientes había sido reinternado o fallecido previamente a la primer visita. Los autores plantean que hubiera sido conveniente estratificar a los pacientes, y visitar más precozmente a los grupos de riesgo.

En relación a la generalización y aplicación, este estudio se llevó a cabo en un centro de alta complejidad, similar a otros estudios publicados. Sin embargo, la intervención no requiere de una logística sofisticada, por lo que los resultados serían extrapolables a otros centros, adaptando los programas para solucionar problemas propios de cada sistema de salud y cada comunidad.

*Ver glosario

\section{Dr. Daniel Ferrante \\ Cardiólogo. Clínica y Maternidad Suizo Argentina.}

